\title{
Stability of a Class of Nonlinear Neutral Stochastic Differential Equations with Variable Time Delays
}

\author{
Meng Wu, Nanjing Huang and Changwen Zhao
}

\begin{abstract}
In this paper, we study the mean square asymptotic stability of a class of generalized nonlinear neutral stochastic differential equations with variable time delays by using fixed point theory. An asymptotic mean square stability theorem with a necessary and sufficient condition is proved which improves and generalizes some well-known results. Finally, two examples are given to illustrate our results.
\end{abstract}

\section{Introduction}

It is well know that stochastic differential equation plays a very important role in formulation and analysis in mechanical, electrical, control engineering, neural network, economic and social sciences. Stochastic delay differential equation, also known as stochastic functional differential equation, is a natural generalization of stochastic ordinary differential equation by allowing the coefficients to depend on the past values. Recently, the studies of stochastic differential equations have attracted the considerable attentions of scholars. Many interesting results concerned with stochastic differential equations have

\footnotetext{
Key Words: fixed point, stability, neutral stochastic differential equations, variable delays.

2010 Mathematics Subject Classification: Primary 34K50; Secondary 34K20.

Received: August, 2010.

Revised: May, 2011.

Accepted: February, 2012.
} 
been obtained over the last few years (see, for example, $[13,19]$ and the references therein).

Liapunov's direct method has been successfully used to investigate stability problems in deterministic/stochastic differential equations and functional differential equations for more than one hundred years. However, there are many difficulties encountered in the study of stability by means of Liapunov's direct method. Recently, Burton and other scholars studied the stability for deterministic systems by using fixed point theory which overcame the difficulties encountered in the study of stability by means of Liapunov's direct method (see, for example, $[2,3,4,5,6,7,8,9,10,14,20,23,24]$ and the references therein).

Very recently, many scholars have began to deal with the stability of stochastic delay differential equations by using fixed point theory (see, for example, [1, 2, 14, 15, 16, 17, 18, 21, 22]). More precisely, Appleby in [1] (also see [2], pp.315-328) considered the almost sure stability for some classical equations by splitting the stochastic differential equation into two equations, one is a fixed stochastic problem and the other is a deterministic stability problem with forcing function. Luo [14] studied the mean square asymptotic stability for a class of linear scalar neutral stochastic differential equations by means of fixed point theory. Furthermore, Luo [15, 16], Luo and Taniguchi [18] used fixed point theory to study the exponential stability of mild solutions of stochastic partial differential equations with bounded delays and with infinite delays. Wu et al. [21, 22] applied fixed point theory to study the stability of a general linear neutral stochastic differential equation and a half-linear neutral stochastic differential equation with variable delays respectively. Luo [17] investigated the exponential stability for the classical stochastic Volterra-Levin equations by using fixed point theory.

Motivated by the previous works mentioned above, in this paper, we study the mean square asymptotic stability of a nonlinear neutral stochastic differential equation with variable delays by applying fixed point theory. An asymptotic mean square stability theorem with a necessary and sufficient condition is proved. Two examples are given to illustrate our results. The results presented in this paper improve and generalize the main results in [3], [14], [23] and [24].

\section{Main Results}

Let $\left(\Omega, \mathcal{F},\left\{\mathcal{F}_{t}\right\}_{t \geq 0}, P\right)$ be a complete filtered probability space and $W(t)$ denote a one-dimensional standard Brownian motion defined on $\left(\Omega, \mathcal{F},\left\{\mathcal{F}_{t}\right\}_{t \geq 0}, P\right)$ such that $\left\{\mathcal{F}_{t}\right\}_{t \geq 0}$ is the natural filtration of $W(t)$. Let $a(t), b(t), \bar{b}(t), c(t), e(t)$, 
$q(t) \in C\left(R^{+}, R\right)$ and $\tau(t), \delta(t) \in C\left(R^{+}, R^{+}\right)$with $t-\tau(t) \rightarrow \infty$ and $t-\delta(t) \rightarrow$ $\infty$ as $t \rightarrow \infty$. Here $C\left(S_{1}, S_{2}\right)$ denotes the set of all continuous function $\phi: S_{1} \rightarrow S_{2}$ with the supremum norm $\|\cdot\|$.

Burton [3] and Zhang [23] studied the equation

$$
x^{\prime}(t)=-\bar{b}(t) x(t-\tau(t))
$$

and proved the following theorems.

Theorem A. (Burton [3]) Suppose that $\tau(t)=r$ and there exists a constant $\alpha<1$ such that

$$
\int_{t-r}^{t}|\bar{b}(s+r)| d s+\int_{0}^{t}|\bar{b}(s+r)| e^{-\int_{s}^{t} \bar{b}(u+r) d u} \int_{s-r}^{s}|\bar{b}(u+r)| d u d s \leq \alpha
$$

for all $t \geq 0$ and $\int_{0}^{\infty} \bar{b}(s) d s=\infty$. Then, for every continuous initial function $\phi:[-r, 0] \rightarrow R$, the solution $x(t)=x(t, 0, \phi)$ of $(1)$ is bounded and tends to zero as $t \rightarrow \infty$.

Theorem B. (Zhang [23]) Suppose that $\tau$ is differentiable, the inverse function $g(t)$ of $t-\tau(t)$ exists, and there exists a constant $\alpha \in(0,1)$ such that for $t \geq 0, \liminf _{t \rightarrow \infty} \int_{0}^{t} \bar{b}(g(s)) d s>-\infty$ and

$$
\begin{aligned}
& \int_{t-\tau(t)}^{t}|\bar{b}(g(s))| d s+\int_{0}^{t} e^{-\int_{s}^{t} \bar{b}(g(u)) d u}|\bar{b}(s)|\left|\tau^{\prime}(s)\right| d s \\
& \quad+\int_{0}^{t} e^{-\int_{s}^{t} \bar{b}(g(u)) d u}|\bar{b}(g(s))| \int_{s-\tau(s)}^{s}|\bar{b}(g(v))| d v d s \leq \alpha<1 .
\end{aligned}
$$

Then the zero solution of (1) is asymptotically stable if and only if $\int_{0}^{t} \bar{b}(g(s)) d s$ $\rightarrow \infty$, as $t \rightarrow \infty$.

Obviously, Theorem B improves Theorem A. Recently, Zhang [24] studied the following half-linear equation

$$
x^{\prime}(t)=-a(t) x(t)+b(t) g(x(t-\tau(t)))
$$

where $g: R \rightarrow R$ is continuous and obtained Theorem C.

Theorem C. (Zhang [24]) Suppose that $\tau(t) \geq 0$ such that for $t \geq 0$, $t-\tau(t) \rightarrow \infty$ as $t \rightarrow \infty$, and there exists a constant $L>0$, for $|x|,|y| \leq L$, $|g(x)-g(y)| \leq|x-y|$ and $q(0)=0$. For $t>0, \liminf _{t \rightarrow \infty} \int_{0}^{t} a(s) d s>-\infty$ and

$$
\sup _{t \geq 0} \int_{0}^{t} e^{-\int_{s}^{t} a(u) d u}|b(s)| d s<1 .
$$

Then the zero solution of (3) is asymptotically stable if and only if $\int_{0}^{t} a(s) d s \rightarrow$ $\infty$, as $t \rightarrow \infty$. 
Recently, Luo [14] considered a linear neutral stochastic differential equation

$$
\begin{aligned}
d[x(t)-q(t) x(t-\tau(t))]= & {[a(t) x(t)+b(t) x(t-\tau(t))] d t } \\
& +[c(t) x(t)+e(t) x(t-\delta(t))] d W(t)
\end{aligned}
$$

and obtained Theorem D.

Theorem D. (Luo [14]) Let $\tau(t)$ be differentiable. Assume that there exists a constant $\alpha \in(0,1)$ and a continuous function $h(t):[0, \infty) \rightarrow R$ such that for $t \geq 0, \liminf _{t \rightarrow \infty} \int_{0}^{t} h(s) d s>-\infty$ and

$$
\begin{aligned}
& |q(t)|+\int_{0}^{t} e^{-\int_{s}^{t} h(u) d u}|h(s)| \int_{s-\tau(s)}^{s}|a(u)+h(u)| d u d s \\
& \quad+\int_{0}^{t} e^{-\int_{s}^{t} h(u) d u} \mid(a(s-\tau(s))+h(s-\tau(s)))\left(1-\tau^{\prime}(s)\right) \\
& \quad+b(s)-q(s) h(s)\left|d s+\int_{t-\tau(t)}^{t}\right| a(s)+h(s) \mid d s \\
& \quad+\left(\int_{0}^{t} e^{-2 \int_{s}^{t} h(u) d u}(|c(s)|+|e(s)|)^{2} d s\right)^{\frac{1}{2}} \leq \alpha<1 .
\end{aligned}
$$

Then the zero solution of (5) is mean square asymptotically stable if and only if $\int_{0}^{t} h(s) d s \rightarrow \infty$, as $t \rightarrow \infty$.

Very recently, Wu et al. [21, 22] generalized Theorems B, C, and D to a general linear neutral stochastic differential equation and to a half-linear neutral stochastic differential equation, respectively. In general, time delay and system uncertainty are commonly encountered and are often sources of instability (see [12]). Thus, it should be interesting to consider the nonlinear stochastic differential equation and study the stability of nonlinear stochastic differential equation with variable time delays.

In this paper, we consider a class of nonlinear neutral stochastic differential equations,

$$
\begin{aligned}
d[x(t)-k(t, x(t-\tau(t))]= & {[a(t) x(t)+f(t, x(t), x(t-\tau(t)))] d t } \\
& +[c(t) x(t)+g(t, x(t), x(t-\delta(t)))] d W(t)(6)
\end{aligned}
$$

with the initial condition

$$
x(s)=\phi(s) \text { for } s \in[m(0), 0],
$$

where $f, g:[0, \infty) \times R \times R \rightarrow R$ and $k:[0, \infty) \times R \rightarrow R$ are continuous, $\phi \in C([m(0), 0], R), x:[m(0), \infty) \times \Omega \rightarrow R$ and

$$
m(0)=\min \{\inf \{s-\tau(s), s \geq 0\}, \inf \{s-\delta(s), s \geq 0\}\} \leq 0 .
$$


If $f(t, x(t), x(t-\tau(t)))=b(t) x(t-\tau(t)), g(t, x(t), x(t-\delta(t)))=e(t) x(t-$ $\delta(t)), k(t, x(t-\tau(t))=q(t) x(t-\tau(t))$, then it is easy to see that (6) reduces to (5). Thus, (6) includes (1), (3) and (5) as special cases. And so, the main aim of this paper is to generalize Theorems B, C and D to apply to (6).

For any $\phi \in C([m(0), 0], R)$, we define $\|\phi\|=\sup _{s \in[m(0), 0]}|\phi(s)|$. For each $\lambda>0$, we define $C(\lambda):=\{\phi \in C([m(0), 0], R):\|\phi\| \leq \lambda\}$. Denote by $F$ the Banach space of all $\mathcal{F}$-adapted processes $\psi(t, \omega):[m(0), \infty) \times \Omega \rightarrow R$ which are almost surely continuous in $t$ with norm

$$
\|\psi\|_{F}=\left\{E\left(\sup _{s \geq m(0)}|\psi(s, \omega)|^{2}\right)\right\}^{\frac{1}{2}} .
$$

Further, we define $F(\lambda)=\left\{\psi \in F:\|\psi\|_{F} \leq \lambda\right\}$ for each $\lambda>0$. Let $\|\psi\|_{F}^{[r, t]}=$ $\left\{E\left(\sup _{s \in[r, t]}|\psi(s, \omega)|^{2}\right)\right\}^{\frac{1}{2}}$ for $r<t$. Then $\|\psi\|_{F}^{[0, \infty)}=\left\{E\left(\sup _{s \geq 0}|\psi(s, \omega)|^{2}\right)\right\}^{\frac{1}{2}}$.

Theorem 2.1. Suppose that $\tau$ is differentiable, and there exist continuous functions $h(t):[0, \infty) \rightarrow R, l(t), m(t), n(t):[0, \infty) \rightarrow R^{+}$and constants $L>0, \alpha \in(0,1)$ such that

(i) $\liminf t \rightarrow \infty \int_{0}^{t} h(s) d s>-\infty$;

(ii) for any $t \geq 0$,

$$
\begin{aligned}
n(t) & +\int_{0}^{t} e^{-\int_{s}^{t} h(u) d u}|h(s)| \int_{s-\tau(s)}^{s}|a(u)+h(u)| d u d s \\
+ & \int_{0}^{t} e^{-\int_{s}^{t} h(u) d u}\left(\left|(a(s-\tau(s))+h(s-\tau(s)))\left(1-\tau^{\prime}(s)\right)\right|\right. \\
& +l(s)+m(s)+|h(s)| n(s)) d s+\int_{t-\tau(t)}^{t}|a(s)+h(s)| d s \\
& +2\left(\int_{0}^{t} e^{-2 \int_{s}^{t} h(u) d u}(|c(s)|+l(s)+m(s))^{2} d s\right)^{\frac{1}{2}} \leq \alpha<1 ;
\end{aligned}
$$

(iii) for any $t \geq 0$,

$$
|f(t, x, y)-f(t, \bar{x}, \bar{y})| \bigvee|g(t, x, y)-g(t, \bar{x}, \bar{y})| \leq l(t)|x-\bar{x}|+m(t)|y-\bar{y}|
$$

and

$$
|k(t, x)-k(t, \bar{x})| \leq n(t)|x-\bar{x}|
$$

for all $x, \bar{x}, y, \bar{y} \in F(L)$ with $f(t, 0,0)=g(t, 0,0)=k(t, 0)=0$. 
Then the zero solution of (6) is mean square asymptotically stable if and only if

$$
\int_{0}^{t} h(s) d s \rightarrow \infty \quad \text { as } t \rightarrow \infty .
$$

Proof. At first, we suppose that (7) holds. Choose $\delta>0, \delta<L$ such that $2 \delta K+\alpha L \leq L$, where $K=\sup _{t \geq 0}\left\{e^{-\int_{0}^{t} h(s) d s}\right\}$. Let $\phi \in C(\delta)$ and set

$$
\begin{array}{r}
S=\{x:[m(0), \infty) \times \Omega \rightarrow R \mid x(t, \omega)=\phi(t) \text { for } t \in[m(0), 0], \\
\left.x(t, \omega) \in F(L) \text { for } t \geq 0, E|x(t, \omega)|^{2} \rightarrow 0 \text { as } t \rightarrow \infty\right\} .
\end{array}
$$

Then it is easy to check that $S$ is a closed subset of $F$. From the definitions of $\|\cdot\|$ and $\|\cdot\|_{F}$, for any $x \in S$ and $t>0$,

$$
\|x\|_{F}=\max \left\{\|\phi\|,\|x\|_{F}^{[0, \infty)}\right\} \leq L .
$$

Define an operator $P: S \rightarrow S$ by $(P x)(t)=\phi(t)$ for $t \in[m(0), 0]$ and for $t \geq 0$,

$$
\begin{aligned}
& (P x)(t) \\
= & \left(\phi(0)-k(0, \phi(-\tau(0)))-\int_{-\tau(0)}^{0}(a(s)+h(s)) \phi(s) d s\right) e^{-\int_{0}^{t} h(s) d s} \\
& +k(t, x(t-\tau(t)))+\int_{t-\tau(t)}^{t}(a(s)+h(s)) x(s) d s \\
& +\int_{0}^{t} e^{-\int_{s}^{t} h(u) d u}\left((a(s-\tau(s))+h(s-\tau(s)))\left(1-\tau^{\prime}(s)\right) x(s-\tau(s))\right. \\
& \quad+f(s, x(s), x(s-\tau(s)))-h(s) k(s, x(s-\tau(s)))) d s \\
& -\int_{0}^{t} e^{-\int_{s}^{t} h(u) d u} h(s)\left(\int_{s-\tau(s)}^{s}(a(u)+h(u)) x(u) d u\right) d s \\
& +\int_{0}^{t} e^{-\int_{s}^{t} h(u) d u}(c(s) x(s)+g(s, x(s), x(s-\delta(s)))) d W(s) \\
:= & \sum_{i=1}^{5} I_{i}(t) .
\end{aligned}
$$

Now, we show the mean square continuity of $P$ on $[0, \infty)$. Let $x \in S, T_{1}>0$ and $|r|$ be sufficiently small. Then

$$
E\left|(P x)\left(T_{1}+r\right)-(P x)\left(T_{1}\right)\right|^{2} \leq 5 \sum_{i=1}^{5} E\left|I_{i}\left(T_{1}+r\right)-I_{i}\left(T_{1}\right)\right|^{2} .
$$


It is easy to verify that

$$
E\left|I_{i}\left(T_{1}+r\right)-I_{i}\left(T_{1}\right)\right|^{2} \rightarrow 0, \quad \text { as } r \rightarrow 0, \quad i=1,2,3,4
$$

From the last term $I_{5}$ in (9), we have

$$
\begin{array}{rl} 
& E\left|I_{5}\left(T_{1}+r\right)-I_{5}\left(T_{1}\right)\right|^{2} \\
=E & E \mid \int_{0}^{T_{1}} e^{-\int_{s}^{T_{1}} h(u) d u}\left(e^{-\int_{T_{1}}^{T_{1}+r} h(u) d u}-1\right) \\
& \cdot(c(s) x(s)+g(s, x(s), x(s-\delta(s)))) d W(s) \\
+ & \left.\int_{T_{1}}^{T_{1}+r} e^{-\int_{s}^{T_{1}+r} h(u) d u}(c(s) x(s)+g(s, x(s), x(s-\delta(s)))) d W(s)\right|^{2} \\
\leq \quad 2 E & \int_{0}^{T_{1}} e^{-2 \int_{s}^{T_{1}} h(u) d u}\left|e^{-\int_{T_{1}}^{T_{1}+r} h(u) d u}-1\right|^{2} \\
& \cdot|c(s) x(s)+g(s, x(s), x(s-\delta(s)))|^{2} d s \\
+ & 2 E \int_{T_{1}}^{T_{1}+r} e^{-2 \int_{s}^{T_{1}+r} h(u) d u}|c(s) x(s)+g(s, x(s), x(s-\delta(s)))|^{2} d s \\
\rightarrow \quad 0, & \text { as } r \rightarrow 0 .
\end{array}
$$

Therefore, $P$ is mean square continuous on $[0, \infty)$.

Next, we verify that $\|P x\|_{F} \leq L$. As $\phi \in C(\delta)$ and $x \in S$,

$$
\begin{aligned}
\|P x\|_{F}^{[0, \infty)} & =\left\{E\left(\sup _{s \geq 0}|P x(s)|^{2}\right)\right\}^{\frac{1}{2}}=\left\{E\left(\sup _{s \geq 0}\left|\sum_{i=1}^{5} I_{i}(s)\right|^{2}\right)\right\}^{\frac{1}{2}} \\
& \leq \sum_{i=1}^{5}\left\{E\left(\sup _{s \geq 0}\left|I_{i}(s)\right|^{2}\right)\right\}^{\frac{1}{2}}
\end{aligned}
$$

By condition $($ iii $)$, we have

$$
|f(t, x, y)| \bigvee|g(t, x, y)| \leq l(t)|x|+m(t)|y| \quad \text { and } \quad|k(t, x)| \leq n(t)|x|
$$

for all $x, y \in F(L)$. It follows from (9), (10), (11), condition (ii) and Doob's 
$L^{p}$-inequality (see [11]) that

$$
\begin{aligned}
& \|P x\|_{F}^{[0, \infty)} \\
& \leq \sup _{s \geq 0}\left\{e^{-\int_{0}^{s} h(v) d v}\right\} \cdot(|\phi(0)|+n(0) \cdot|\phi(-\tau(0))| \\
& \left.+\int_{-\tau(0)}^{0}|a(v)+h(v)| \cdot|\phi(v)| d v\right) \\
& +\left(E \sup _{s \geq 0}\left(n(s) \cdot|x(s-\tau(s))|+\int_{s-\tau(s)}^{s}|a(v)+h(v)| \cdot|x(v)| d v\right)^{2}\right)^{\frac{1}{2}} \\
& +\left(E \operatorname { s u p } _ { s \geq 0 } \left(\int _ { 0 } ^ { s } e ^ { - \int _ { v } ^ { s } h ( u ) d u } \left(\left|(a(v-\tau(v))+h(v-\tau(v)))\left(1-\tau^{\prime}(v)\right)\right|\right.\right.\right. \\
& \left.\cdot|x(v-\tau(v))|+|f(v, x(v), x(v-\delta(v)))|+|h(v)||k(v, x(v-\tau(v)))|) d v)^{2}\right)^{\frac{1}{2}} \\
& +\left(E \sup _{s \geq 0}\left(\int_{0}^{s} e^{-\int_{v}^{s} h(u) d u}|h(v)|\left(\int_{v-\tau(v)}^{v}|a(u)+h(u)| \cdot|x(u)| d u\right) d v\right)^{2}\right)^{\frac{1}{2}} \\
& +2 \sup _{s \geq 0}\left(E \int_{0}^{s} e^{-2 \int_{v}^{s} h(u) d u}(|c(v)| \cdot|x(v)|+|g(v, x(v), x(v-\delta(v)))|)^{2} d v\right)^{\frac{1}{2}} \\
& \leq \delta K\left(1+n(0)+\int_{-\tau(0)}^{0}|a(v)+h(v)| d v\right) \\
& +\|x\|_{F} \cdot \sup _{s \geq 0}\left\{n(s)+\int_{s-\tau(s)}^{s}|a(v)+h(v)| d v+\int_{0}^{s} e^{-\int_{v}^{s} h(u) d u}\right. \\
& \cdot\left(\left|(a(v-\tau(v))+h(v-\tau(v)))\left(1-\tau^{\prime}(v)\right)\right|+l(v)+m(v)+|h(v)| n(v)\right) d v \\
& +\int_{0}^{s} e^{-\int_{v}^{s} h(u) d u}|h(v)|\left(\int_{v-\tau(v)}^{v}|a(u)+h(u)| d u\right) d v \\
& \left.+2\left(\int_{0}^{s} e^{-2 \int_{v}^{s} h(u) d u}(|c(v)|+l(v)+m(v))^{2} d v\right)^{\frac{1}{2}}\right\} \\
& \leq 2 \delta K+\alpha L \leq L \text {. }
\end{aligned}
$$

Further, from (8), we have

$$
\|P x\|_{F}=\max \left\{\|\phi\|,\|P x\|_{F}^{[0, \infty)}\right\} \leq L .
$$

Thirdly, we verify that $E|(P x)(t)|^{2} \rightarrow 0$ as $t \rightarrow \infty$. Since $E|x(t)|^{2} \rightarrow 0$, $t-\delta(t) \rightarrow \infty$ as $t \rightarrow \infty$, for each $\epsilon>0$, there exists a $T_{1}>0$ such that $s \geq T_{1}$ implies $E|x(s)|^{2}<\epsilon$ and $E|x(s-\delta(s))|^{2}<\epsilon$. By condition $(i i)$, for $t \geq T_{1}$, 
the last term $I_{5}$ in (9) satisfies

$$
\begin{aligned}
& E\left|I_{5}(t)\right|^{2} \\
\leq & E \int_{0}^{T_{1}} e^{-2 \int_{s}^{t} h(u) d u}(|c(s)| \cdot|x(s)|+l(s)|x(s)|+m(s)|x(s-\delta(s))|)^{2} d s \\
& +E \int_{T_{1}}^{t} e^{-2 \int_{s}^{t} h(u) d u}(|c(s)| \cdot|x(s)|+l(s)|x(s)|+m(s)|x(s-\delta(s))|)^{2} d s \\
\leq & \left(\|x\|_{F}^{\left[m(0), T_{1}\right]}\right)^{2} \int_{0}^{T_{1}} e^{-2 \int_{s}^{t} h(u) d u}(|c(s)|+l(s)+m(s))^{2} d s+\alpha \epsilon \\
\leq & \|x\|_{F}^{2} \cdot e^{-2 \int_{T_{1}}^{t} h(u) d u} \int_{0}^{T_{1}} e^{-2 \int_{s}^{T_{1}} h(u) d u}(|c(s)|+l(s)+m(s))^{2} d s+\alpha \epsilon \\
\leq & L^{2} \alpha^{2} e^{-2 \int_{T_{1}}^{t} h(u) d u}+\alpha \epsilon .
\end{aligned}
$$

From (7), there exists $T_{2}>T_{1}$ such that $L^{2} \alpha^{2} e^{-2 \int_{T_{1}}^{t} h(u) d u}<\epsilon$ for $t \geq T_{2}$. Thus, for $t \geq T_{2}, E\left|I_{5}(t)\right|^{2}<\epsilon+\alpha \epsilon$. This proves that $E\left|I_{5}(t)\right|^{2} \rightarrow 0$, as $t \rightarrow \infty$. Similarly, we can show that $E\left|I_{i}(t)\right|^{2} \rightarrow 0, i=1,2,3,4$, as $t \rightarrow \infty$. Thus, $E|(P x)(t)|^{2} \rightarrow 0$ as $t \rightarrow \infty$. Hence $P x \in S$.

Now we show that $P: S \rightarrow S$ is a contraction mapping. For any $x, y \in S$, we have

$$
\begin{aligned}
& \|P x-P y\|_{F} \\
= & \left(E \sup _{s \geq m(0)}|(P x)(s)-(P y)(s)|^{2}\right)^{\frac{1}{2}} \\
= & \left(E \sup _{s \geq 0} \mid k(s, x(s-\tau(s)))-k(s, y(s-\tau(s)))\right. \\
+ & \int_{s-\tau(s)}^{s}(a(v)+h(v))(x(v)-y(v)) d v+\int_{0}^{s} e^{-\int_{v}^{s} h(u) d u}((a(v-\tau(v)) \\
& +h(v-\tau(v)))\left(1-\tau^{\prime}(v)\right)(x(v-\tau(v))-y(v-\tau(v))) \\
& +f(v, x(v), x(v-\tau(v)))-f(v, y(v), y(v-\tau(v))) \\
& -h(v)(k(v, x(v-\tau(v)))-k(v, y(v-\tau(v))))) d v \\
- & \int_{0}^{s} e^{-\int_{v}^{s} h(u) d u} h(v)\left(\int_{v-\tau(v)}^{v}(a(u)+h(u))(x(u)-y(u)) d u\right) d v \\
+ & \int_{0}^{s} e^{-\int_{v}^{s} h(u) d u}(c(v)(x(v)-y(v))+g(v, x(v), x(v-\tau(v))) \\
& \left.\quad-g(v, y(v), y(v-\tau(v))))\left.d W(v)\right|^{2}\right)^{\frac{1}{2}}
\end{aligned}
$$




$$
\begin{aligned}
\leq \quad & \|x-y\|_{F} \cdot \sup _{s \geq 0}\left\{n(s)+\int_{s-\tau(s)}^{s}|a(v)+h(v)| d v\right. \\
& +\int_{0}^{s} e^{-\int_{v}^{s} h(u) d u}|h(v)| \int_{v-\tau(v)}^{v}|a(u)+h(u)| d u d v+\int_{0}^{s} e^{-\int_{v}^{s} h(u) d u} \\
& \cdot\left(\left|(a(v-\tau(v))+h(v-\tau(v)))\left(1-\tau^{\prime}(v)\right)\right|+l(v)+m(v)+|h(v)| n(v)\right) d v \\
& \left.+2\left(\int_{0}^{s} e^{-2 \int_{v}^{s} h(u) d u}(|c(v)|+l(v)+m(v))^{2} d v\right)^{\frac{1}{2}}\right\} \\
\leq \quad & \alpha\|x-y\|_{F} .
\end{aligned}
$$

Therefore, $P: S \rightarrow S$ is contraction mapping and so $P$ has a fixed point $x \in S$, which is a solution of (6) with $x(s)=\phi(s)$ on $[m(0), 0]$ and $E|x(t)|^{2} \rightarrow 0$ as $t \rightarrow \infty$.

To obtain the mean square asymptotic stability, we need to show that the zero solution of (6) is mean square stable. From $($ ii $)$, we can choose $\Delta>0$ such that $\alpha^{2}+\Delta<1$. Thus, we can find a constant $N>0$ such that

$$
\begin{aligned}
& \left(1+\frac{1}{N}\right)\left(n(t)+\int_{0}^{t} e^{-\int_{s}^{t} h(u) d u}|h(s)| \int_{s-\tau(s)}^{s}|a(u)+h(u)| d u d s\right. \\
& +\int_{t-\tau(t)}^{t}|a(s)+h(s)| d s+\int_{0}^{t} e^{-\int_{s}^{t} h(u) d u} \\
& \left.\cdot\left(\left|(a(s-\tau(s))+h(s-\tau(s)))\left(1-\tau^{\prime}(s)\right)\right|+l(s)+m(s)+|h(s)| n(s)\right) d s\right)^{2} \\
& +4(1+N) \int_{0}^{t} e^{-2 \int_{s}^{t} h(u) d u}(|c(s)|+l(s)+m(s))^{2} d s \leq \alpha^{2}+\Delta<1 .
\end{aligned}
$$

Let $\epsilon>0$ and $\epsilon<L$ be given and choose $\delta_{0}>0$ and $\delta_{0}<\epsilon$ satisfying the following condition

$$
4(1+N) \delta_{0}^{2} K^{2}+\left(\alpha^{2}+\Delta\right) \epsilon<\epsilon
$$

where $N$ is defined in (12). If $x(t)=x(t, 0, \phi)$ is a solution of (6) with $\|\phi\|<\delta_{0}$, then $x(t)=(P x)(t)$ which is defined in (9). We claim that $E|x(t)|^{2}<\epsilon$ for all $t \geq 0$. Notice that $x(t)=\phi(t)$ for $t \in[m(0), 0]$, so $E|x(t)|^{2},\|\phi(t)\|^{2}<\epsilon$ for $t \in[m(0), 0]$. If there exists $t^{*}>0$ such that $E\left|x\left(t^{*}\right)\right|^{2}=\epsilon$ and $E|x(t)|^{2}<\epsilon$ 
for $t \in\left[m(0), t^{*}\right)$, then (9) and (12) imply that

$$
\begin{aligned}
& E\left|x\left(t^{*}\right)\right|^{2} \\
\leq & (1+N)\|\phi\|^{2}\left(1+n(0)+\int_{-\tau(0)}^{0}|a(s)+h(s)| d s\right)^{2} e^{-2 \int_{0}^{t^{*}} h(u) d u} \\
& +\epsilon\left(1+\frac{1}{N}\right)\left(\left|q\left(t^{*}\right)\right|+\int_{0}^{t^{*}} e^{-\int_{s}^{t^{*}} h(u) d u}\left(\int_{s-\tau(s)}^{s}|a(u)+h(u)| d u\right)|h(s)| d s\right. \\
& +\int_{t^{*}-\tau\left(t^{*}\right)}^{t^{*}}|a(s)+h(s)| d s+\int_{0}^{t^{*}} e^{-\int_{s}^{t^{*}} h(u) d u} \\
& \left.\cdot\left(\left|(a(s-\tau(s))+h(s-\tau(s)))\left(1-\tau^{\prime}(s)\right)\right|+l(s)+m(s)+|h(s)| n(s)\right) d s\right)^{2} \\
& +\epsilon \int_{0}^{t^{*}} e^{-2 \int_{s}^{t^{*}} h(u) d u}(|c(s)|+l(s)+m(s))^{2} d s \\
\leq \quad & (1+N) \delta_{0}^{2}\left(1+n(0)+\int_{-\tau(0)}^{0}|a(s)+h(s)| d s\right)^{2} e^{-2 \int_{0}^{t^{*}} h(u) d u}+\left(\alpha^{2}+\Delta\right) \epsilon \\
< & \epsilon,
\end{aligned}
$$

which contradicts the definition of $t^{*}$. Thus, the zero solution of (6) is mean square asymptotically stable if (7) holds.

Conversely, we suppose that (7) fails. From condition $(i)$, there exists a sequence $\left\{t_{n}\right\}$ with $t_{n} \rightarrow \infty$ as $n \rightarrow \infty$ such that $\lim _{n \rightarrow \infty} \int_{0}^{t_{n}} h(u) d u=\zeta$ for some $\zeta \in R$. Then, we can choose a constant $J>0$ satisfying $\int_{0}^{t_{n}} h(u) d u \in[-J, J]$ for all $n \geq 1$. Denote

$$
\begin{aligned}
\omega(s):=\quad & \left|(a(s-\tau(s))+h(s-\tau(s)))\left(1-\tau^{\prime}(s)\right)\right| \\
& +l(s)+m(s)+|h(s)| n(s)+|h(s)| \int_{s-\tau(s)}^{s}|a(u)+h(u)| d u
\end{aligned}
$$

for all $s \geq 0$. From condition $(i i)$, we have

$$
\int_{0}^{t_{n}} e^{-\int_{s}^{t_{n}} h(u) d u} \omega(s) d s \leq \alpha
$$

which implies

$$
\int_{0}^{t_{n}} e^{\int_{0}^{s} h(u) d u} \omega(s) d s \leq \alpha e^{\int_{0}^{t_{n}} h(u) d u} \leq e^{J} .
$$

Therefore, the sequence $\left\{\int_{0}^{t_{n}} e^{\int_{0}^{s} h(u) d u} \omega(s) d s\right\}$ has a convergent subsequence. 
Without loss of generality, we can assume that $\lim _{n \rightarrow \infty} \int_{0}^{t_{n}} e^{\int_{0}^{s} h(u) d u} \omega(s) d s=$ $\gamma$ for some $\gamma>0$. Let $m$ be an integer such that

$$
\int_{t_{m}}^{t_{n}} e^{\int_{0}^{s} h(u) d u} \omega(s) d s<\frac{\delta_{1}}{8 K}
$$

for all $n \geq m$, where $0<\delta_{1}<1$ satisfies $8 \delta_{1}^{2} K^{2} e^{2 J}+\left(\alpha^{2}+\Delta\right)<1$.

Now, we consider the solution $x(t)=x\left(t, t_{m}, \phi\right)$ of $(6)$ with $\left\|\phi\left(t_{m}\right)\right\|=\delta_{1}$ and $\|\phi(t)\|<\delta_{1}$ for $t<t_{m}$. By the similar method in (13), we have $E|x(t)|^{2}<$ 1 for $t \geq t_{m}$. We may choose $\phi$ so that

$$
\begin{aligned}
G\left(t_{m}\right):=\phi\left(t_{m}\right)-k\left(t_{m}, \phi\left(t_{m}-\tau\left(t_{m}\right)\right)\right) & \\
& \quad-\int_{t_{m}-\tau\left(t_{m}\right)}^{t_{m}}(a(s)+h(s)) \phi(s) d s \geq \frac{\delta_{1}}{2} .
\end{aligned}
$$

It follows from (9), (14) and (15) with $x(t)=(P x)(t)$ that for $n \geq m$,

$$
\begin{aligned}
& E\left|x\left(t_{n}\right)-k\left(t_{n}, x\left(t_{n}-\tau\left(t_{n}\right)\right)\right)-\int_{t_{n}-\tau\left(t_{n}\right)}^{t_{n}}(a(s)+h(s)) x(s) d s\right|^{2} \\
\geq & G^{2}\left(t_{m}\right) e^{-2 \int_{t_{m}}^{t_{n}} h(u) d u}-2 G\left(t_{m}\right) e^{-\int_{t_{m}}^{t_{n}} h(u) d u} \int_{t_{m}}^{t_{n}} e^{-\int_{s}^{t_{n}} h(u) d u} \omega(s) d s \\
\geq & G\left(t_{m}\right) e^{-2 \int_{t_{m}}^{t_{n}} h(u) d u}\left(G\left(t_{m}\right)-2 e^{-\int_{0}^{t_{m}} h(u) d u} \int_{t_{m}}^{t_{n}} e^{\int_{0}^{s} h(u) d u} \omega(s) d s\right) \\
\geq & \frac{\delta_{1}}{2} e^{-2 \int_{t_{m}}^{t_{n}} h(u) d u}\left(\frac{\delta_{1}}{2}-2 K \int_{t_{m}}^{t_{n}} e^{\int_{0}^{s} h(u) d u} \omega(s) d s\right) \geq \frac{\delta_{1}^{2}}{8} e^{-2 J}>0 .(16)
\end{aligned}
$$

If the zero solution of (6) is mean square asymptotic stable, then $E|x(t)|^{2}=$ $E\left|x\left(t, t_{m}, \phi\right)\right|^{2} \rightarrow 0$ as $t \rightarrow \infty$. Since $t_{n}-\tau\left(t_{n}\right) \rightarrow \infty, t_{n}-\delta\left(t_{n}\right) \rightarrow \infty$ as $n \rightarrow \infty$ and conditions (ii) and (iii) hold, then

$$
E\left|x\left(t_{n}\right)-k\left(t_{n}, x\left(t_{n}-\tau\left(t_{n}\right)\right)\right)-\int_{t_{n}-\tau\left(t_{n}\right)}^{t_{n}}(a(s)+h(s)) x(s) d s\right|^{2} \rightarrow 0,
$$

as $n \rightarrow \infty$ which contradicts (16). Thus, (7) is necessary for Theorem 2.1. This completes the proof.

Remark 2.1. Theorem 2.1 is still true if condition (ii) is satisfied for $t \geq t_{a}$ with some $t_{a} \in R^{+}$. 
Remark 2.2. The method in this paper can be extended to the following nonlinear neutral stochastic differential equation with several variable delays:

$$
\begin{gathered}
d\left(x(t)-\sum_{i=1}^{n} k_{i}\left(t, x\left(t-\tau_{i}(t)\right)\right)\right. \\
=\left(a(t) x(t)+\sum_{i=1}^{n} f_{i}\left(t, x(t), x\left(t-\tau_{i}(t)\right)\right)\right) d t \\
\quad+\left(c(t) x(t)+\sum_{j=1}^{m} g_{j}\left(t, x(t), x\left(t-\delta_{j}(t)\right)\right)\right) d W(t) .
\end{gathered}
$$

Choosing $h(t) \equiv-a(t)$ in Theorem 2.1, we have the following result.

Corollary 2.1. Suppose that $\tau$ is differential, and there exist continuous functions $l(t), m(t), n(t):[0, \infty) \rightarrow R^{+}$and constants $L>0, \alpha \in(0,1)$ such that

$\left(i^{\prime}\right) \liminf _{t \rightarrow \infty} \int_{0}^{t}-a(s) d s>-\infty$;

$\left(i i^{\prime}\right)$ for any $t \geq 0$,

$$
\begin{aligned}
n(t) & +\int_{0}^{t} e^{\int_{s}^{t} a(u) d u}(l(s)+m(s)+|a(s)| n(s)) d s \\
& +2\left(\int_{0}^{t} e^{2 \int_{s}^{t} a(u) d u}(|c(s)|+l(s)+m(s))^{2} d s\right)^{\frac{1}{2}} \leq \alpha<1 ;
\end{aligned}
$$

(iii') for any $t \geq 0$,

$$
|f(t, x, y)-f(t, \bar{x}, \bar{y})| \bigvee|g(t, x, y)-g(t, \bar{x}, \bar{y})| \leq l(t)|x-\bar{x}|+m(t)|y-\bar{y}|
$$

and

$$
|k(t, x)-k(t, \bar{x})| \leq n(t)|x-\bar{x}|
$$

for all $x, \bar{x}, y, \bar{y} \in F(L)$ with $f(t, 0,0)=g(t, 0,0)=k(t, 0)=0$.

Then the zero solution of (6) is mean square asymptotically stable if and only if $\int_{0}^{t} a(s) d s \rightarrow \infty$ as $t \rightarrow \infty$.

Now, we consider a special case of nonlinear neutral stochastic differential equation (6) that

$$
\begin{aligned}
& d[x(t)-k(t, x(t-\tau(t))] \\
= & {[a(t) x(t)+b(t) x(t-\tau(t))+f(t, x(t), x(t-\tau(t)))] d t } \\
& +[c(t) x(t)+e(t) x(t-\delta(t))+g(t, x(t), x(t-\delta(t)))] d W(t),
\end{aligned}
$$


Note that (17) reduces to (5) when $f(t, x(t), x(t-\tau(t))) \equiv g(t, x(t), x(t-$ $\delta(t))) \equiv 0$ and $k(t, x(t-\tau(t))=q(t) x(t-\tau(t))$. Then, we have the following results.

Theorem 2.2. Suppose that $\tau$ is differentiable, and there exist continuous functions $h(t):[0, \infty) \rightarrow R, l(t), m(t), n(t):[0, \infty) \rightarrow R^{+}$and constants $L>0, \alpha \in(0,1)$ such that

$\left(i^{\circ}\right) \liminf _{t \rightarrow \infty} \int_{0}^{t} h(s) d s>-\infty$;

(ii $\left.{ }^{\circ}\right)$ for any $t \geq 0$,

$$
\begin{aligned}
n(t) & +\int_{0}^{t} e^{-\int_{s}^{t} h(u) d u}|h(s)| \int_{s-\tau(s)}^{s}|a(u)+h(u)| d u d s \\
& +\int_{t-\tau(t)}^{t}|a(s)+h(s)| d s+\int_{0}^{t} e^{-\int_{s}^{t} h(u) d u}(l(s)+m(s)+|h(s)| n(s) \\
& \left.+\left|(a(s-\tau(s))+h(s-\tau(s)))\left(1-\tau^{\prime}(s)\right)+b(s)\right|\right) d s \\
& +2\left(\int_{0}^{t} e^{-2 \int_{s}^{t} h(u) d u}(|c(s)|+|e(s)|+l(s)+m(s))^{2} d s\right)^{\frac{1}{2}} \leq \alpha<1
\end{aligned}
$$

$\left(i i i^{\circ}\right)$ for any $t \geq 0$,

$$
\begin{aligned}
& |f(t, x, y)-f(t, \bar{x}, \bar{y})| \bigvee|g(t, x, y)-g(t, \bar{x}, \bar{y})| \leq l(t)|x-\bar{x}|+m(t)|y-\bar{y}| \\
& \text { and } \\
& \qquad|k(t, x)-k(t, \bar{x})| \leq n(t)|x-\bar{x}| \\
& \text { for all } x, \bar{x}, y, \bar{y} \in F(L) \text { with } f(t, 0,0)=g(t, 0,0)=k(t, 0)=0
\end{aligned}
$$

Then the zero solution of (17) is mean square asymptotically stable if and only if $\int_{0}^{t} h(s) d s \rightarrow \infty$ as $t \rightarrow \infty$.

The proof is analogous to that of Theorem 2.1 and so we omit it here.

Remark 2.3. Theorem 2.2 improves Theorem D under different conditions.

Let $h(t) \equiv-b(p(t))$ in Theorem 2.2. Then we have the following corollary.

Corollary 2.2. Suppose that $\tau$ is differential, the inverse function $p(t)$ of $t-\tau(t)$ exists, and there exist continuous functions $l(t), m(t), n(t):[0, \infty) \rightarrow$ $R^{+}$and constants $L>0, \alpha \in(0,1)$ such that

$\left(i^{*}\right) \liminf _{t \rightarrow \infty} \int_{0}^{t}-b(p(s)) d s>-\infty$; 
$\left(i i^{*}\right)$ for any $t \geq 0$

$$
\begin{aligned}
& n(t)+\int_{0}^{t} e^{\int_{s}^{t} b(p(u)) d u}|b(p(s))| \int_{s-\tau(s)}^{s}|a(u)-b(p(u))| d u d s \\
& +\int_{t-\tau(t)}^{t}|a(s)-b(p(s))| d s+\int_{0}^{t} e^{\int_{s}^{t} b(p(u)) d u} \cdot(l(s)+m(s) \\
& \left.\quad+|b(p(s))| n(s)+\left|(a(s-\tau(s))-b(s))\left(1-\tau^{\prime}(s)\right)+b(s)\right|\right) d s \\
& \quad+2\left(\int_{0}^{t} e^{2 \int_{s}^{t} b(p(u)) d u}(|c(s)|+|e(s)|+l(s)+m(s))^{2} d s\right)^{\frac{1}{2}} \leq \alpha<1 ;
\end{aligned}
$$

$\left(i i i^{*}\right)$ for any $t \geq 0$,

$$
|f(t, x, y)-f(t, \bar{x}, \bar{y})| \bigvee|g(t, x, y)-g(t, \bar{x}, \bar{y})| \leq l(t)|x-\bar{x}|+m(t)|y-\bar{y}|
$$

and

$$
|k(t, x)-k(t, \bar{x})| \leq n(t)|x-\bar{x}|
$$

for all $x, \bar{x}, y, \bar{y} \in F(L)$ with $f(t, 0,0)=g(t, 0,0)=k(t, 0)=0$.

Then the zero solution of (17) is mean square asymptotically stable if and only if $\int_{0}^{t} b(p(s)) d s \rightarrow \infty$ as $t \rightarrow \infty$.

Remark 2.4. When $k(t, x(t-\tau(t))) \equiv f(t, x(t), x(t-\tau(t))) \equiv g(t, x(t), x(t-$ $\delta(t))) \equiv a(t) \equiv c(t) \equiv e(t) \equiv 0$ and $b(t) \equiv-\bar{b}(t)$, we know that Corollary 2.2 still holds if the condition ( $\left.i i^{*}\right)$ is replaced by (2). Therefore, Corollary 2.2 is a generalization of Theorem B.

Now, we consider another special case of nonlinear neutral stochastic differential equation (6) that

$$
d x(t)=-a(t) x(t)+f(t, x(t), x(t-\tau(t))) d t,
$$

Note that (18) reduces to (3) when $f(t, x(t), x(t-\tau(t))) \equiv b(t) g(x(t-\tau(t)))$. Then, we have the following result.

Theorem 2.3. Suppose that $\tau$ is differential, and there exist continuous functions $h(t):[0, \infty) \rightarrow R, l(t), m(t):[0, \infty) \rightarrow R^{+}$and constants $L>0$, $\alpha \in(0,1)$ such that

$\left(i^{\star}\right) \liminf _{t \rightarrow \infty} \int_{0}^{t} h(s) d s>-\infty$; 
$\left(i i^{\star}\right)$ for any $t \geq 0$,

$$
\begin{aligned}
& \int_{0}^{t} e^{-\int_{s}^{t} h(u) d u}|h(s)| \int_{s-\tau(s)}^{s}|h(u)-a(u)| d u d s \\
& \quad+\int_{t-\tau(t)}^{t}|h(s)-a(s)| d s+\int_{0}^{t} e^{-\int_{s}^{t} h(u) d u}(l(s)+m(s) \\
& \left.\quad+\left|(h(s-\tau(s))-a(s-\tau(s)))\left(1-\tau^{\prime}(s)\right)\right|\right) d s \leq \alpha<1 ;
\end{aligned}
$$

$\left(i i i^{\star}\right)$ for any $t \geq 0$,

$$
|f(t, x, y)-f(t, \bar{x}, \bar{y})| \leq l(t)|x-\bar{x}|+m(t)|y-\bar{y}|
$$

for all $x, \bar{x}, y, \bar{y} \in F(L)$ with $f(t, 0,0)=0$.

Then the zero solution of (18) is asymptotically stable if and only if $\int_{0}^{t} h(s) d s \rightarrow$ $\infty$ as $t \rightarrow \infty$.

The proof is analogous to that of Theorem 2.1 and so we omit it here.

Remark 2.5. When $f(t, x(t), x(t-\tau(t))) \equiv b(t) g(x(t-\tau(t)))$, choosing $h(t) \equiv$ $a(t), l(t) \equiv 0$ and $m(t) \equiv|b(t)|$, Corollary 2.3 reduces to Theorem $C$.

\section{Two Examples}

In this section, we give two examples to illustrate the applications of our main results.

Example 3.1. Consider the following nonlinear neutral stochastic delay differential equation

$$
\begin{aligned}
& d\left(x(t)-\frac{1}{8} x^{2}\left(\frac{3 t}{4}\right)\right) \\
= & \left(-2 x(t)+\frac{e^{-2 t}}{8} \sin \left(x(t)+x\left(\frac{3 t}{4}\right)\right) \cdot \cos \left(x(t)-x\left(\frac{3 t}{4}\right)\right)\right) d t \\
+ & \left(\frac{1}{9} x(t)+\frac{e^{-t}}{7} \cos \left(x(t)+x\left(\frac{t}{2}\right)\right) \cdot \sin \left(x(t)-x\left(\frac{t}{2}\right)\right)\right) d W(t) .
\end{aligned}
$$

Then the zero solution of (19) is mean square asymptotically stable. 
Proof. Let

$$
\begin{aligned}
f(t, x(t), x(t-\tau(t)) & :=\frac{e^{-2 t}}{8} \sin \left(x(t)+x\left(t-\frac{t}{4}\right)\right) \cdot \cos \left(x(t)-x\left(t-\frac{t}{4}\right)\right), \\
g(t, x(t), x(t-\delta(t)) & :=\frac{e^{-t}}{7} \cos \left(x(t)+x\left(t-\frac{t}{2}\right)\right) \cdot \sin \left(x(t)-x\left(t-\frac{t}{2}\right)\right) .
\end{aligned}
$$

Since $|\sin x| \leq|x|$ for $x \in R$, the we get

$$
\begin{aligned}
& \mid f(t, x(t), x(t-\tau(t))-f(t, y(t), y(t-\tau(t)) \mid \\
& \quad \leq \frac{e^{-2 t}}{8}|x(t)-y(t)|+\frac{e^{-2 t}}{8}\left|x\left(t-\frac{t}{4}\right)-y\left(t-\frac{t}{4}\right)\right| \\
& \mid g(t, x(t), x(t-\delta(t))-g(t, y(t), y(t-\delta(t)) \mid \\
& \quad \leq \frac{e^{-t}}{7}|x(t)-y(t)|+\frac{e^{-t}}{7}\left|x\left(t-\frac{t}{2}\right)-y\left(t-\frac{t}{2}\right)\right|
\end{aligned}
$$

As $\left|x^{2}\right| \leq|x|$ when $|x| \leq 1$, we can choose $L=1 / 2, l(t)=m(t)=e^{-t} / 7$ and $n(t)=1 / 8$ such that the condition $($ iii $)$ of Theorem 2.1 holds. Moreover, it is easy to verify that $t-\tau(t)=t-t / 4 \rightarrow \infty$ and $t-\delta(t)=t-t / 2 \rightarrow \infty$ as $t \rightarrow \infty$.

Choosing $h(t)=2$ in Theorem 2.1, we have

$$
\begin{gathered}
\int_{0}^{t} e^{-\int_{s}^{t} h(u) d u}\left(\left|(a(s-\tau(s))+h(s-\tau(s)))\left(1-\tau^{\prime}(s)\right)\right|+l(s)+m(s)\right. \\
+|h(s)| n(s)) d s=\int_{0}^{t} e^{-2(t-s)}\left(\frac{2 e^{-s}}{7}+\frac{1}{4}\right) d s \leq 0.18 \\
2\left(\int_{0}^{t} e^{-2 \int_{s}^{t} h(u) d u}(|c(s)|+l(s)+m(s))^{2} d s\right)^{\frac{1}{2}} \\
=2\left(\int_{0}^{t} e^{-2(t-s)}\left(\frac{2 e^{-s}}{7}+\frac{1}{9}\right) d s\right)^{\frac{1}{2}} \leq 0.68
\end{gathered}
$$

and

$\int_{t-\tau(t)}^{t}|a(s)+h(s)| d s=\int_{0}^{t} e^{-\int_{s}^{t} h(u) d u}|h(s)| \int_{s-\tau(s)}^{s}|a(u)+h(u)| d u d s=0$.

It easy to check that $\int_{0}^{t} h(s) d s \rightarrow \infty$ as $t \rightarrow \infty$. Let $\alpha=1 / 8+0.18+0.68$. Then $\alpha=0.985<1$ and so the zero solution of (19) is mean square asymptotically stable.

Example 3.2. Consider the following delay differential equation

$$
x^{\prime}(t)=-\frac{1}{5} x(t)+2 e^{-t} \sin \left(\frac{1}{10} x\left(t-e^{-t}\right)\right) .
$$


Then the zero solution of (20) is asymptotically stable.

Proof. Let

$$
f\left(t, x(t), x(t-\tau(t)):=2 e^{-t} \sin \left(\frac{1}{10} x\left(t-e^{-t}\right)\right) .\right.
$$

Since $\left|\sin \frac{x}{10}\right| \leq \frac{1}{10}|x|$ for $x \in R$, we have

$\mid f\left(t, x(t), x(t-\tau(t))-f\left(t, y(t), y(t-\tau(t))\left|\leq \frac{e^{-t}}{5}\right| x\left(t-e^{-t}\right)-y\left(t-e^{-t}\right) \mid\right.\right.$.

Therefore, we can choose $l(t) \equiv 0, m(t)=e^{-t} / 5$ and $L$ for any positive constant such that the condition $\left(i i i^{\star}\right)$ of Theorem 2.3 holds. Moreover, it is easy to verify that $t-\tau(t)=t-e^{-t} \rightarrow \infty$ as $t \rightarrow \infty$. Choosing $h(t) \equiv 0.3$ in Theorem 2.3, we have

$$
\begin{aligned}
& \int_{t-\tau(t)}^{t}|h(s)-a(s)| d s=\int_{t-e^{-t}}^{t}(0.3-0.2) d s \rightarrow 0.1 e^{-t} \leq 0.1 \\
& \int_{0}^{t} e^{-\int_{s}^{t} h(u) d u}|h(s)| \int_{s-\tau(s)}^{s}|h(u)-a(u)| d u d s \leq \int_{0}^{t} \frac{0.03}{e^{0.3(t-s)}} d s \leq 0.1
\end{aligned}
$$

and

$$
\begin{aligned}
& \int_{0}^{t} e^{-\int_{s}^{t} h(u) d u}\left(\left|(h(s-\tau(s))-a(s-\tau(s)))\left(1-\tau^{\prime}(s)\right)\right|+l(s)+m(s)\right) d s \\
= & \int_{0}^{t} e^{-0.3(t-s)}\left[(0.3-0.2)\left(1+e^{-s}\right)+\frac{e^{-s}}{5}\right] d s \leq 0.36 .
\end{aligned}
$$

It is easy to see that all conditions of Theorem 2.3 hold for $\alpha=0.1+0.1+$ $0.36=0.56<1$. Thus, Theorem 2.3 implies that the zero solution of $(20)$ is asymptotically stable.

However, Theorem $\mathrm{C}$ can not be used to verify that the zero solution of (20) is asymptotically stable. In fact, noticing that $\left|\sin \frac{x}{10}-\sin \frac{y}{10}\right| \leq|x-y|$ for all $x, y \in R, b(t) \equiv 2 e^{-t}, a(t) \equiv 1 / 5$ and

$$
\int_{0}^{t} e^{-\int_{s}^{t} a(u) d u}|b(s)| d s=\int_{0}^{t} e^{-0.2(t-s)} \cdot 2 e^{-s} d s<1.33 .
$$

Obviously, the condition (4) of Theorem $\mathrm{C}$ does not hold with $\alpha=1.33>$ 1. 


\section{Acknowledgement}

This work was supported by the National Natural Science Foundation of China (71101099, 11171237) and the Fundamental Research Funds for the Central Universities (2009SCU11096).

\section{References}

[1] J.A.D. Appleby, Fixed points, stability and harmless stochastic perturbations, preprint.

[2] T.A. Burton, Stability by Fixed Point Theory for Functional Differential Equations, Dover Publications, Inc., New York, 2006.

[3] T.A. Burton, Stability by fixed point theory or Liapunov's theory: a comparison, Fixed Point Theory, 4 (2003), 15-32.

[4] T.A. Burton, Liapunov functionals, fixed points, and stability by Krasnoselskii's theorem, Nonlinear Stud., 9 (2001), 181-190.

[5] T.A. Burton, Fixed points and stability of a nonconvolution equation, Proc. Amer. Math. Soc., 132 (2004), 3679-3687.

[6] T.A. Burton and T. Furumochi, Fixed points and problems in stability theory for ordinary and functional differential equations, Dynamical Systems and Appl., 10 (2001), 89-116.

[7] T.A. Burton and T. Furumochi, Krasnoselskii's fixed point theorem and stability, Nonlinear Anal. TMA, 49 (2002), 445-454.

[8] T.A. Burton and B. Zhang, Fixed points and stability of an integral equation: Nonuniquess, Appl. Math. Lett., 17 (2004), 893-846.

[9] C.H. Jin and J.W. Luo, Fixed points and stability in neutral differential equations with variable delays, Proc. Amer. Math. Soc., 136 (2008), 909918.

[10] S.M. Jung, A fixed point approach to the stability of differential equations $y^{\prime}=F(x, y)$, Bull. Malays. Math. Sci. Soc., 33(1) (2010), 47-56.

[11] I. Karatzas and S.E. Shreve, Brownian Motion and Stochastic Calculus, 2nd ed. Springer, New York, 1991.

[12] V.B. Kolmanovskii and V.R. Nosov, Stability of Functional Differential Equations, Academic Press, New York, 1986. 
[13] K. Liu, Stability of Infinite Dimensional Stochastic Differential Equation with Applications, Chapman \& Hall/CRC, Boca Raton, 2006.

[14] J.W. Luo, Fixed points and stability of neutral stochastic delay differential equations, J. Math. Anal. Appl., 334 (2007), 431-440.

[15] J.W. Luo, Fixed points and exponential stability of mild solutions of stochastic partial differential equations with delays, J. Math. Anal. Appl., 342 (2008), 753-760.

[16] J.W. Luo, Stability of stochastic partial differential equations with infinite delays, J. Comput. Appl. Math., 222 (2008), 364-371.

[17] J.W. Luo, Fixed points and exponential stability for stochastic VolterraLevin equations, J. Comput. Appl. Math., 234 (2010), 934-940.

[18] J.W. Luo and T. Taniguchi, Fixed points and stability of stochastic neutral partial differential equations with infinite delays, Stoch. Anal. Appl., 27 (2009), 1163-1173.

[19] X.R. Mao, Stochastic Differential Equations and their Applications, Horwood Publ. House, Chichester, 1997.

[20] Y.N. Raffoul, Stability in neutral nonlinear differential equations with functional delays using fixed-point theory, Math. Comput. Modelling, 40 (2004), 691-700.

[21] M. Wu, N.J. Huang and C.W. Zhao, Stability of half-linear neutral stochastic differential equations with delays, Bull. Austral. Math. Soc. 80 (2009), 369-383.

[22] M. Wu, N.J. Huang and C.W. Zhao, Fixed points and stability in neutral stochastic differential equations with variable eelays, Fixed Point Theory Appl., 2008 (2008), Article ID 407352.

[23] B. Zhang, Fixed points and stability in differential equations with variable delays, Nonlinear Anal., 63 (2005), e233-e242.

[24] B. Zhang, Contraction mapping and stability in a delay-differential equation, Proc. Dynam. Sys. Appl., 4 (2004), 183-190.

Meng $\mathrm{Wu}$

College of Business Administration,

Sichuan University,

Chengdu, Sichuan 610064, China.

Email: wumeng@scu.edu.cn, shancherish@hotmail.com 
STABILITY OF A CLASS OF NONLINEAR NEUTRAL STOCHASTIC

Nanjing Huang,

Department of Mathematics,

Sichuan University,

Chengdu, Sichuan 610064, China.

Email: njhuang@scu.edu.cn

Changwen Zhao,

College of Business Administration,

Sichuan University,

Chengdu, Sichuan 610064, China.

Email: cwzhao@scu.edu.cn 
